\title{
Clinical Significance of Low Signal Intensity Area Surrounding Stent Struts Identified by Optical Coherence Tomography
}

\author{
Kohei Ishibashi, ${ }^{1}$ MD, Atsushi Tanaka, ${ }^{1}$ MD, Hironori Kitabata, ${ }^{1}$ MD, Takashi Kubo, ${ }^{1}$ MD, \\ Manabu Kashiwagi, ${ }^{1}$ MD, Kenichi KomuKaI, ${ }^{1}$ MD, Yasushi Ino, ${ }^{1}$ MD, Takashi Tanimoto, ${ }^{1}$ MD, \\ Kazushi Takemoto, ${ }^{1}$ MT, Shigeho Takarada, ${ }^{1}$ MD, Kumiko Hirata, ${ }^{1}$ MD, Masato Mizukoshi, ${ }^{1}$ MD, \\ Toshio Imanishi, ${ }^{1} \mathrm{MD}$, and Takashi AKasaKa, ${ }^{1} \mathrm{MD}$
}

\begin{abstract}
SUMMARY
Previous intravascular ultrasound studies have shown that echolucent neointimal hyperplasia occasionally appears after bare-metal stent (BMS) or sirolimus-eluting stent (SES) implantation. Optical coherence tomography (OCT) studies have also demonstrated that paclitaxel-eluting stent (PES) restenosis exhibited similar images showing low signal intensity areas (LSIA) surrounding stent struts and three-layer appearance (TLA). The aim of the present study was to investigate the clinical significance of LSIA on OCT images in various types of stents. Fifty nine consecutive patients who underwent scheduled follow-up coronary angiography and OCT were enrolled. There was no significant difference in the prevalence of LSIA among the 3 stent groups (BMS 30\%, SES 19\%, PES 28\%, $P=0.70$ ). LSIA thickness was larger in the PES group than in the other stent groups (BMS $0.51 \pm 0.21 \mathrm{~mm}$, SES $0.35 \pm 0.06 \mathrm{~mm}$, PES $0.87 \pm 0.19 \mathrm{~mm}, P<$ 0.01). The ratio of LSIA thickness to the neointimal thickness was also larger in PES compared with other stents (BMS $53 \pm 9 \%$, SES $57 \pm 8 \%$, PES $77 \pm 5 \%, P<0.01$ ). Also, LSIA thickness in patients with in-stent restenosis (ISR) was significantly larger than in those without ISR $(0.37 \pm 0.37 \mathrm{~mm}$ versus $0.12 \pm 0.26 \mathrm{~mm}, P=0.048)$. Our results suggest that LSIA might be involved in excessive neointimal formation, and that the healing response after PES implantation might be different from BMS or SES. (Int Heart J 2013; 54: 7-10)
\end{abstract}

Key words: In-stent restenosis

$\mathrm{T}$ he recent development of intravascular optical coherence tomography (OCT) has provided a high-resolution $(10-20 \mu \mathrm{m})$ imaging method for plaque characterization. ${ }^{1-5)}$ Histological studies have shown that OCT can identify microstructures in atherosclerotic plaques in vivo., ${ }^{6,7}$

Previous intravascular ultrasound (IVUS) studies have shown that echolucent neointimal hyperplasia "Black holes" occasionally appear, irrespective of stent type, after stent implantation. ${ }^{8-12)}$ A recent OCT study also reported a very similar figure, namely a three-layer appearance (TLA), in paclitaxeleluting stent (PES) restenosis. ${ }^{13)}$ It is highlighted as an image showing a low signal intensity area (LSIA) surrounding stent struts on OCT images. However, the clinical significance of LSIA is still unclear. The aim of this study was to investigate the clinical significance of LSIA in various types of stent using OCT.

\section{MethodS}

Study population: A study population of 59 consecutive patients who met the following criteria was enrolled; 1) Patients who were admitted to Wakayama Medical University between
April 2007 and November 2008, 2) Patients who underwent single stent implantation for a de novo native coronary artery lesion with standard techniques during this period, and 3) $\mathrm{Pa}$ tients who agreed to receiving both scheduled follow-up coronary angiography (CAG) and OCT at 9 months after percutaneous coronary intervention (PCI). Our exclusion criteria were presentation of congestive heart failure (Ejection fraction < $40 \%$ ) and chronic renal failure (Creatinine $>1.5 \mathrm{mg} / \mathrm{dL}$ ). A sirolimus-eluting stent (SES) (Cypher ${ }^{\mathrm{TM}}$, Cordis Corp., Miami Lakes, FL, USA) was implanted in 21 patients, PES (Taxus Express $^{\mathrm{TM}}$, Boston Scientific Corp., Natick, MA, USA) in 18 patients, and a bare-metal stent (BMS) (Duraflex, Goodman Co., Ltd., Nagoya, Japan) in 20 patients. Demographic and clinical data were collected prospectively. All patients underwent scheduled follow-up CAG and OCT at 9 months after stent implantation and OCT data for these 59 patients was analyzed. Written informed consent was obtained from all patients for participation in this study.

Angiographic analysis: Scheduled follow-up CAG was performed using a 5-Fr. Judkins-type catheter via the femoral approach. All patients received an intravenous bolus injection of $2000 \mathrm{IU}$ heparin and intracoronary isosorbide dinitrate $(2 \mathrm{mg})$ before CAG. The stented site was identified on the basis of

From the ${ }^{1}$ Department of Cardiovascular Medicine, Wakayama Medical University, Wakayama, Japan.

Address for correspondence: Takashi Kubo, MD, Department of Cardiovascular Medicine, Wakayama Medical University, 811-1 Kimiidera, Wakayama, Wakayama 641-8510, Japan.

Received for publication June 27, 2012.

Revised and accepted October 19, 2012 
findings on CAG. Coronary angiograms were reviewed separately by 2 independent observers (HK and TT) unaware of the OCT findings. Binary in-stent restenosis (ISR) was defined as $\geqq 50 \%$ diameter stenosis in the in-segment area (including the stent area and 5-mm segments proximal and distal to the stent edges). Target lesion revascularization (TLR) was defined as any revascularization procedure, percutaneous or surgical, involving the target lesion and performed in the presence of angiographic restenosis accompanied by symptoms or signs of ischemia.

OCT procedure and image interpretation: After completion of follow-up CAG, OCT was used to observe the postintervention site of the coronary artery. A 0.016-inch OCT catheter (Imagewire, LightLab imaging, Inc., Westford, MA, USA) was advanced to the distal end of the postintervention site through a 3-Fr. (distal) occlusion balloon catheter $\left(\right.$ Helios $^{\circledR}$, Goodman Co., Ltd., Nagoya, Japan). If the lesion presented with severe tortuosity, severe stenosis, or a heavy calcium burden, we first advanced a conventional PCI guidewire (0.014-inch) across the lesion before exchanging it for the OCT Imagewire using an occlusion balloon catheter. To remove blood cells from the imaging field, the occlusion balloon was inflated to $0.5 \mathrm{~atm}$ proximal to the postintervention site, and lactated Ringer's solution was infused into the coronary artery from the distal tip of the occlusion balloon catheter at a rate of $0.5 \mathrm{~mL} / \mathrm{s}$.

For proximal lesions, we used a continuous-flushing (nonocclusive) technique of OCT image acquisition, which is a newly developed alternative to the balloon-occlusion technique. ${ }^{14)}$ To flush the vessel, we infused a mixture of commercially available dextran 40 and lactated Ringer's solution (lowmolecular-weight Dextran L Injection, Otsuka Pharmaceutical Factory, Tokushima, Japan) directly from the guiding catheter at a rate of 2.5 to $4.5 \mathrm{~mL} / \mathrm{s}$ with an injector pump (Mark V, Medrad Inc, Warrendale, PA, USA). Regardless of the OCT technique used, in all cases, the postintervention site was imaged with an automatic pullback device traveling at $1 \mathrm{~mm} / \mathrm{s}$. The OCT images were digitalized and analyzed by an $\mathrm{M} 2 \mathrm{CV}$ OCT console. All OCT images were analyzed by 2 independent investigators (KI and AT) who were blinded to the clinical presentations. When there was discordance between observers, a consensus reading was obtained.

The definition of the presence of TLA was as follows: 1) The inner luminal layer appeared concentric, homogenous, and signal-rich, 2) A second layer consisting of a low-reflective area with poorly delineated borders followed, and 3) The third layer was in direct contact above the stent struts and demonstrated only minimal signal intensity. The second and third layers were defined as LSIA. Representative OCT images of TLA in each stent are shown in the Figure. The quantitative OCT analysis of neointima was performed at the minimal lumen area site which was selected by visual screening. The thickest part of the neointima was also determined visually to measure the thickness of neointima and LISA. A stent strut was considered suitable for analysis only if it had a well-defined bright "blooming" appearance. Neointimal thickness was defined as the distance between the center of the stent strut blooming and luminal surface. LISA thickness was defined as the distance between the center of the stent strut blooming and LISA contour. The ratio of LSIA thickness to the neointimal thickness was calculated by dividing LSIA thickness by neointimal thickness. Representative OCT images of neointima in the
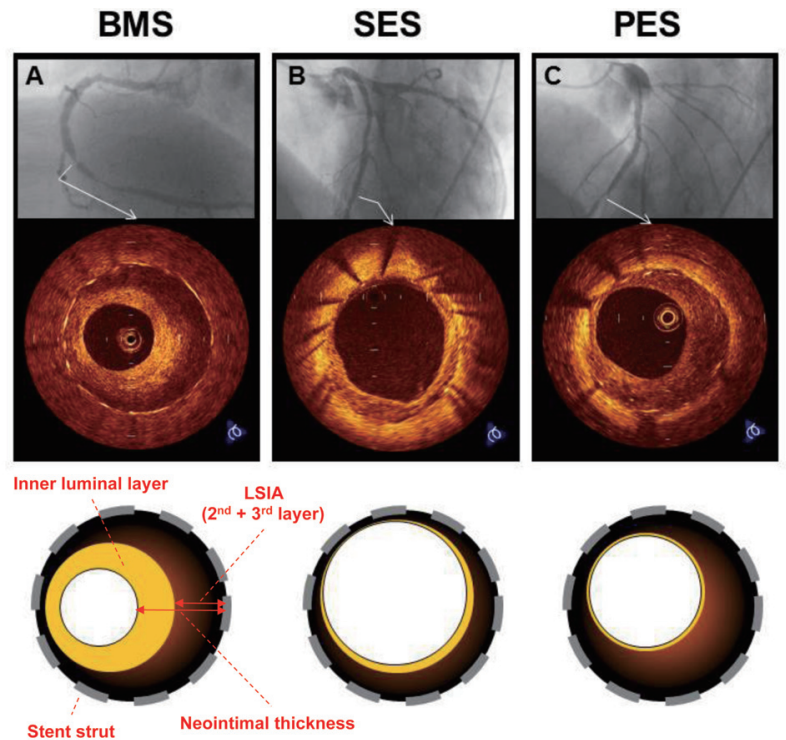

Figure. Representative OCT images of neointima in the stented segments. Angiography and OCT imaging were performed at 9 months after stent implantation. BMS had a thick inner luminal layer and LSIA (A). SES contained a thin inner luminal layer and LSIA (B). PES included an extremely thin inner luminal layer and thick LSIA $(\mathbf{C})$. The ratio of LSIA thickness to neointimal thickness was greater in PES than in BMS and SES. BMS indicates bare metal stent; LSIA, low signal intensity area; OCT, optical coherence tomography; PES, paclitaxel-eluting stent; and SES, sirolimus-eluting stent.

stented segments are shown in the Figure.

Statistical analysis: Statistical analysis was performed using StatView 5.0 J (SAS Institute Inc., Cary, NC, USA). Results are expressed as the mean \pm SD for continuous variables. Categorical data are presented as numbers $(\%)$. Continuous variables were compared using Student's $t$ test. Categorical data were compared by the chi-square test or Fisher's exact test, as appropriate. To control for multiple comparisons, pairwise tests (Scheffe' method) of incidence were performed if the contingency table for all 3 stent groups was significant. A $P$ value $<0.05$ was considered statistically significant.

\section{Results}

Both follow-up CAG and OCT were completed in all patients and the corresponding images were obtained in all 59 patients. Patient characteristics are presented in Table I. There were no differences in terms of age, gender, classic coronary risk factors except for hypertension, target vessel, stent length, or maximum inflation pressure among the 3 stent groups (BMS, SES, and PES). The stent size was significantly larger in BMS than in the other stents (BMS $3.3 \pm 0.4 \mathrm{~mm}$, SES 2.9 $\pm 0.3 \mathrm{~mm}$, PES $2.8 \pm 0.2 \mathrm{~mm}, P<0.01)$. The frequency of ISR and TLR is presented in Table II. ISR and TLR tended to be lower in the SES group than in the other groups, but there was no significant difference in this cohort.

There was no significant difference in the prevalence of LSIA among the 3 stent groups (BMS 30\%, SES 19\%, PES $28 \%, P=0.70)$. The neointimal thickness, LSIA thickness, and ratio of LSIA thickness to neointimal thickness in each stent 
are presented in Table III. While there were no differences in the neointimal thickness among the 3 stents, LSIA thickness was significantly larger for PES than BMS or SES (BMS 0.51 $\pm 0.21 \mathrm{~mm}$, SES $0.35 \pm 0.06 \mathrm{~mm}$, PES $0.87 \pm 0.19 \mathrm{~mm}, P<$ 0.01). The ratio of LSIA thickness to neointimal thickness was also larger for PES than BMS and SES (BMS $53 \pm 9 \%$, SES $57 \pm 8 \%$, PES $77 \pm 5 \%, P<0.01)$. TLA was observed more frequently in TLR patients than in non-TLR patients $(100 \%$ versus $16 \%, P<0.01)$. Also, LSIA thickness in patients with ISR was significantly larger than in those without ISR $(0.37 \pm$ $0.37 \mathrm{~mm}$ versus $0.12 \pm 0.26 \mathrm{~mm}, P=0.048$ ).

\section{Discussion}

Previous studies have shown that in-stent neointimal hyperplasia of the coronary arteries consists predominantly of spindle-shaped mesenchymal cells (a-actin-positive smooth muscle cells) with very little collagen and elastin. ${ }^{15-18)}$ Until recently, IVUS was used as the standard imaging modality to evaluate neointimal hyperplasia. The corresponding IVUS image for neointimal hyperplasia is hypoechoic but not echolucent. An echolucent appearance of the restenotic tissue is an unusual phenomenon in IVUS imaging. Previous IVUS studies have shown that echolucent neointimal hyperplasia, namely black hole phenomenon, occasionally appears after the implantation of not only drug eluting stents (DES) but also after $\mathrm{BMS}$, and is associated with restenotic lesions. ${ }^{8-12)}$ Very recently, Goto, et al reported that black hole phenomenon in IVUS images was associated predominantly with intimal hyperplasia

Table I. Baseline Characteristics

\begin{tabular}{lcccc}
\hline & BMS & SES & PES & $P$ \\
\hline Number & 20 & 21 & 18 & \\
Age, years & $66 \pm 10$ & $66 \pm 13$ & $70 \pm 9$ & 0.42 \\
Male gender (\%) & $15(75)$ & $16(76)$ & $15(83)$ & 0.80 \\
Diabetes mellitus (\%) & $7(35)$ & $8(38)$ & $9(50)$ & 0.61 \\
Hypertension (\%) & $12(60)$ & $17(81)$ & $17(94)$ & 0.03 \\
Hypercholesterolemia (\%) & $15(75)$ & $15(71)$ & $14(78)$ & 0.90 \\
$\quad$ total cholesterol $>220$ & & & & \\
$\quad$ mg/dL) & & & & \\
Cigarette smoking $(\%)$ & $17(85)$ & $12(57)$ & $13(72)$ & 0.14 \\
Target vessel & & & & 0.38 \\
$\quad$ LAD (\%) & $8(40)$ & $13(62)$ & $7(39)$ & \\
$\quad$ LCx (\%) & $3(15)$ & $2(10)$ & $5(28)$ & \\
$\quad$ RCA (\%) & $9(45)$ & $6(29)$ & $6(33)$ & \\
Stent size, mm & $3.3 \pm 0.4$ & $2.9 \pm 0.3$ & $2.8 \pm 0.2$ & $<0.01$ \\
Stent length, mm & $21 \pm 13$ & $27 \pm 15$ & $21 \pm 5$ & 0.16 \\
Maximum inflation pres- & $14 \pm 3$ & $16 \pm 3$ & $15 \pm 3$ & 0.39 \\
sure, atm & & & & \\
\hline
\end{tabular}

Values are presented as $n(\%)$ or mean \pm standard deviation. LAD indicates left anterior descending coronary artery; $\mathrm{LCx}$, left circumflex artery; and RCA, right coronary artery. Other abbreviations as in the Figure. with hypocellular smooth muscle cells and abundant extracellular matrix such as proteoglycans. ${ }^{19)}$ Therefore, this black hole phenomenon of echolucent tissue is considered to be caused by the fact that the tissue is acellular and necrotic and lacks connective tissue elements. LSIA by OCT represents very similar characteristics as compared to those found with the black hole phenomenon in IVUS. ${ }^{19)}$ In this study, this LSIA image could be observed equally in each stent but to a higher degree in PES. This indicates that this image might represent a common healing pathway after stent implantation irrespective of stent types but is enhanced particularly in PES. In a previous animal study, the greatest extent of inflammation and fibrin deposition in a rabbit model occurred with the PES, then with the SES, and least with the BMS. The greatest amount of neointimal thickness occurred with the PES compared with BMS and SES. ${ }^{20)}$ An autopsy study also reported that PES showed a predominance of fibrin deposition in humans. ${ }^{21)}$ Therefore, we speculate the following processes for LSIA formation. Firstly fibrin may be deposited around the stent struts, and the amount may be especially large for PES. Next, during the healing process after stent implantation, this fibrin may replace acellular matrix such as proteoglycan. While these processes would not be prevented by the antiproliferative effect of DESs, excessive neointimal formation is provoked in cases with LSIA. LSIA is associated with excessive neointimal formation. This scenario raises a possible alternative process for excessive neointimal formation that should be targeted in the development of new stents.

There are some limitations associated with the present study. First, the patient population was relatively small. Second, the study was prospective but not randomized. Third, differences in the frequency of hypertension and stent size among the 3 stent groups may have had an influence on the neointimal characteristics. Fourth, the mechanism of LSIA formation should be investigated in future histopathological studies.

In conclusion, our results suggest that LSIA might be involved in excessive neointimal formation, and that the healing response after PES implantation might be different from other stents.

Table III. Neointimal Thickness, LSIA Thickness, and Ratio of LSIA Thickness to Neointimal Thickness on OCT Findings

\begin{tabular}{lcccr}
\hline & BMS & SES & PES & $P$ \\
\hline Neointimal thickness, mm & $0.97 \pm 0.35$ & $0.62 \pm 0.11$ & $1.12 \pm 0.21$ & 0.06 \\
LSIA thickness, mm & $0.51 \pm 0.21$ & $0.35 \pm 0.06$ & $0.87 \pm 0.19^{* \#}$ & $<0.01$ \\
Ratio of LSIA thickness to the neointimal thickness & $53 \pm 9$ & $57 \pm 8$ & $77 \pm 5^{* *}$ & $<0.01$ \\
\hline
\end{tabular}

Values are presented as mean \pm standard deviation. ${ }^{*} P<0.05$, BMS versus PES; ${ }^{*} P<0.05$, SES versus PES. Abbreviations as in the Figure. 


\section{REFERENCES}

1. Jang IK, Bouma BE, Kang DH, et al. Visualization of coronary atherosclerotic plaques in patients using optical coherence tomography: comparison with intravascular ultrasound. J Am Coll Cardiol 2002; 39: 604-9.

2. Brezinski ME, Tearney GJ, Bouma BE, et al. Optical coherence tomography for optical biopsy. Properties and demonstration of vascular pathology. Circulation 1996; 93: 1206-13.

3. Kume T, Akasaka T, Kawamoto T, et al. Assessment of coronary intima-media thickness by optical coherence tomography: comparison with intravascular ultrasound. Circ J 2005; 69: 903-7.

4. Kume T, Akasaka T, Kawamoto T, et al. Visualization of neointima formation by optical coherence tomography. Int Heart J 2005; 46: 1133-6.

5. Kume T, Akasaka T, Kawamoto T, et al. Assessment of coronary arterial plaque by optical coherence tomography. Am J Cardiol 2006; 97: 1172-5.

6. Jang IK, Tearney GJ, MacNeill B, et al. In vivo characterization of coronary atherosclerotic plaque by use of optical coherence tomography. Circulation 2005; 111: 1551-5.

7. Yabushita H, Bouma BE, Houser SL, et al. Characterization of human atherosclerosis by optical coherence tomography. Circulation 2002; 106: 1640-5.

8. Costa Jde R Jr, Mintz GS, Carlier SG, et al. Frequency and determinations of black holes in sirolimus-eluting stent restenosis. J Invasive Cardiol 2006; 18: 348-52.

9. Dilcher CE, Chan RC, Pregowski J, et al. Dose volume histogram assessment of late stent malapposition after intravascular brachytherapy. Cardiovasc Radiat Med 2002; 3: 190-2.

10. Sianos G, Kay IP, Costa MA, et al. Geographical miss during catheter-based intracoronary beta-radiation: incidence and implications in the BRIE study. J Am Coll Cardiol 2001; 38: 415-20.

11. Castagna MT, Mintz GS, Weissman N, Maehara A, Finet G, Waksman R. "Black hole": echolucent restenotic tissue after brachytherapy. Circulation 2001; 103: 778.

12. Stabile E, Escolar E, Weigold G, et al. Marked malapposition and aneurysm formation after sirolimus-eluting coronary stent implantation. Circulation 2004; 110: e47-8.

13. Tanimoto A, Aoki J, Patrick WS, Evelyn Regar. Paclitaxel-eluting stent restenosis shows three-layer appearance by optical coherence tomography. Euro Interv 2006; 1: 484.

14. Kataiwa H, Tanaka A, Kitabata H, Imanishi T, Akasaka T. Safety and usefulness of non-occlusion image acquisition technique for optical coherence tomography. Circ J 2008; 72: 1536-7.

15. Komatsu R, Ueda M, Naruko T, Kojima A, Becker AE. Neointimal tissue response at sites of coronary stenting in humans: macroscopic, histological, and immunohistochemical analyses. Circulation 1998; 98: 224-33.

16. Farb A, Sangiorgi G, Carter AJ, et al. Pathology of acute and chronic coronary stenting in humans. Circulation 1999; 99: 44-52.

17. Kornowski R, Hong MK, Tio FO, Bramwell O, Wu H, Leon MB. In-stent restenosis: contributions of inflammatory responses and arterial injury to neointimal hyperplasia. J Am Coll Cardiol 1998; 31: 224-30.

18. Carter AJ, Laird JR, Kufs WM, et al. Coronary stenting with a novel stainless steel balloon-expandable stent: determinants of neointimal formation and changes in arterial geometry after placement in an atherosclerotic model. J Am Coll Cardiol 1996; 27 : 1270-7.

19. Goto K, Shiode N, Shirota K, et al. Pathological finding of sirolimus-eluting stent (SES) restenosis lesion with black hole appearance on intravascular ultrasound. Circ J 2009; 73: 1969-71.

20. Nakazawa G, Finn AV, John MC, Kolodgie FD, Virmani R. The significance of preclinical evaluation of sirolimus-, paclitaxel-, and zotarolimus-eluting stents. Am J Cardiol 2007; 100: 36M-44M. (Review)

21. Joner M, Finn AV, Farb A, et al. Pathology of drug-eluting stents in humans: delayed healing and late thrombotic risk. J Am Coll Cardiol 2006; 48: 193-202. 